\title{
An iterative fragment-scheme for the ACKS2 electronic polarization model: Application to molecular dimers and chains
}

\author{
Patrick Gütlein, ${ }^{\dagger}$ Jochen Blumberger, ${ }^{\dagger}, \mathbf{I}$ and Harald Oberhofer ${ }^{*}, \dagger$ \\ $\dagger$ †hair for Theoretical Chemistry and Catalysis Research Center, Technische Universität \\ München, Lichtenbergstrasse 4, D-85747 Garching, Germany \\ $\ddagger$ Department of Physics and Astronomy, University College London, London WC1E 6BT, \\ $U K$. \\ 【Institute for Advanced Study, Technische Universität München, Lichtenbergstrasse 2 a, \\ D-85748 Garching, Germany. \\ E-mail: harald.oberhofer@tum.de
}

\begin{abstract}
The treatment of electrostatic interactions is a key ingredient in the force-field based simulation of condensed phase systems. Most approaches used fixed, site-specific point charges. Yet, it is now clear that many applications of force fields (FFs) demand more sophisticated treatments, prompting the implementation of charge equilibration methods in polarizable FFs to allow the redistribution of charge within the system. One approach allowing both, charge redistribution and site specific polarization, while at the same time solving methodological shortcomings of earlier methods, is the firstprinciples-derived atom condensed Kohn-Sham density functional theory method approximated to second order (ACKS2). In this work we present two fragment approaches
\end{abstract}


to ACKS2, termed f-ACKS2 and a self-consistent version, scf-ACKS, that treat condensed phase systems as a collection of electronically polarizable molecular fragments. The fragmentation approach to ACKS2 not only leads to a more transferable and less system specific collection of electronic response parameters, but also opens up the method to large condensed phase systems. We validate the accuracies of f-ACKS2 and scf-ACKS2 by comparing polarization energies and induced dipole moments for a number of charged hydrocarbon dimers against DFT reference calculations. Finally, we also apply both fragmented ACKS2 variants to calculate the polarization energy for electron-hole pair separation along a chain of anthracene molecules and find excellent agreement with reference DFT calculations.

\section{Introduction}

Nowadays, atomistic or coarse-grained force fields are an essential tool in the materials' modeling community, enabling an efficient sampling of the phase space of condensed phase systems. ${ }^{1-4}$ Whether based on training by ab-initio references or experimental properties, or - recently — machine learned models, force fields allow molecular simulations of systems that are too large for treatment with explicit electronic structure methods. They have been used very successfully for the simulation of biological systems, ${ }^{5-7}$ catalysis, ${ }^{8,9}$ and energy conversion materials like batteries ${ }^{10,11}$ or organic semiconductors. ${ }^{12,13}$

In all cases, the accuracy of force field approaches rests critically on their ability to represent the different types of interaction between atoms or molecules. ${ }^{4,14,15}$ One key ingredient is the accurate description of electrostatic interactions, especially in systems where small changes in the electrostatic potential strongly influence local properties, as, e.g., in

protein folding ${ }^{16}$ or excitation energy and charge transport. ${ }^{17,18}$ Therefore, earlier molecular mechanics simulations frequently employed an inexpensive, but crude classical point charge representation of the mean-field electrostatic interactions. ${ }^{19,20}$ In recent years, though, the static, isotropic nature and lack of directionality of the simple point charge model were high- 
lighted, ${ }^{21-24}$ prompting the development of improved electrostatic models by either adding off-center point charges or higher angular momentum terms like dipole and quadrupole moments. ${ }^{25,26}$ Going further and incorporating the electronic many-body polarization induced by local electrostatic potential changes - e.g. due to dynamical fluctuations of other molecules, presence of excess charge carriers, or external voltages - in a classical interatomic model framework is a difficult task. ${ }^{21,27}$ Indeed, polarization corrections so far exhibited mixed successes compared to well-tuned non-polarizable force field parametrizations. ${ }^{28-31}$ Popular empirical models for the explicit polarization in force fields are the Drude oscillator (or core-shell) $)^{32,33}$ and the atomic inducible dipole (or distributed polarizabilities) approach, ${ }^{34,35}$ which employ a charge attached to the atomic nuclei by a harmonic spring or atom-centered inducible point dipoles, respectively, to capture intra-atomic charge rearrengements. While they are very efficient and easy to implement, they exclude specific atom-atom charge transfer terms and are difficult to parametrize thoroughly, raising the problem of system transferability. ${ }^{36,37}$ On the contrary, the X-Pol ${ }^{38}$ (formerly MODEL ${ }^{39,40}$ ) method combines a simplified semi-empirical quantum mechanical description of a molecule -including its polarization - with the efficient force field representation of the intermolecular interaction potential, to approximately maintain the non-classical nature of electrons. Along similar lines, force field techniques have been combined with quantum chemical calculations of molecular fragments to obtain atomic partial charges and molecular fragment dipole moments on-the-fly (i.e. every few picoseconds for protein simulations). ${ }^{41,42}$ While these approaches show promising accuracy and transferability, e.g. protein solvation in water, ${ }^{43,44}$ they suffer from a much increased computational cost compared to standard force fields. ${ }^{45}$ Finally, in an attempt to capture charge reorganization and polarization yet at the cost of standard force fields, a population of fluctuating atomic charges, initially described by the electronegativity equilibration method (EEM) ${ }^{46}$ has become a widespread choice for the description of electronic polarization. In the three and a half decades since EEM's inception it has undergone many refinements in the form of the charge equilibration (QEq) ${ }^{47}$ chemical 
potential equilibration $(\mathrm{CPE}){ }^{48}$ atom-atom charge transfer $(\mathrm{AACT})^{49}$ and split-charge equilibration (SQE) $)^{50}$ schemes. However, many of these models suffer from overestimation of the long-range charge transfer due to incorrect kinetic energy contributions to the electronic many-body response. A recently suggested method for the calculation of electronic polarizaton, the atom-condensed Kohn-Sham density functional theory approximated to second order (ACKS2), ${ }^{51,52}$ could alleviate these methodological drawbacks.

ACKS2 is a linear electronic response technique derived from first-principles, based on an atom-projected description of the perturbative Kohn-Sham (KS) density functional theory (DFT) electron density rearrangements induced by an external potential. In a recent study, we developed a transferable Cartesian Gaussian basis set representation as first step towards applying ACKS2 as a general electronic polarization contribution in force fields. ${ }^{53}$ We found that ACKS2-calculated induced dipoles and polarization energies of organic molecules due to simple external electrostatic perturbations were in excellent agreement with the results of full DFT calculations. This suggests that ACKS2 could be a promising method for the description of electronic polarization between molecules in the condensed phase. However, so far ACKS2 has only been used to calculate the polarization response to a static external electric field, not between two mutually polarizable entities like molecules. Therefore, in order to make ACKS2 amenable to force field simulations, it is necessary to develop an approach that describes the mutual polarization of molecules within the ACKS2 framework.

In this study, we introduce a fragment-approach to ACKS2 and treat the simulation system as an assembly of polarizable molecular subunits. In this approach the intramolecular and intermolecular polarization energy are accounted for at ACKS2 level under the constraint that the total charge of each fragment is equal to the charge of the unperturbed fragment. Hence, similarly to other polarization models, the method accounts for intramolecular but not intermolecular charge redistribution. In the following section 2 we briefly summarize the main concepts of ACKS2, before we describe in detail our new ACKS2 fragmentation approach. The latter is validated against the perturbative KS-DFT parent method in section 
3 on a set of neutral and charged organic dimers, and illustrated further for a chain of organic molecules carrying an excess electron and electron hole. Finally, concluding remarks are given in section 4.

\section{Theory}

\subsection{Atom-condensed Kohn-Sham DFT approximated to 2nd order}

The atom-condensed Kohn-Sham density functional theory approximated to second order, ${ }^{51,52}$ a recently developed extension to more traditional charge equilibration schemes, ${ }^{48}$ was demonstrated to be an accurate, yet transferable first-principles-based method for the calculation of response properties, like the electronic polarization energies and induced dipole moments. ${ }^{53}$ It describes electronic polarization in the presence of an external potential using a simplified perturbative Kohn-Sham density functional theory approach within the limit of linear response. The first-principles nature of this method has a number of distinct benefits compared to empirical polarization models, in that all parameters of the ACKS2 model have a direct physical meaning and can be derived exactly from DFT calculations. ${ }^{51,53}$ In the interest of brevity, here we only give a brief overview of the method, necessary for the derivation of the fragment scheme. For a more detailed description of the method we refer the reader to references ${ }^{51,52}$ or our previous work. ${ }^{53}$

In ACKS2, the response of the electron density, $\Delta \rho$, and the Kohn-Sham potential, $\Delta v_{\mathrm{KS}}$, to an external potential, $\Delta v_{\text {ext }}$, is expanded in terms of atom centered basis functions $g_{n}\left(h_{m}\right)$ with expansion coefficients $c_{n}\left(d_{m}\right)$,

$$
\begin{aligned}
& \Delta \rho(\mathbf{r})=\sum_{n}^{N} c_{n} g_{n}(\mathbf{r}) \\
& \Delta v_{\mathrm{KS}}(\mathbf{r})=\sum_{m}^{M} d_{m} h_{m}(\mathbf{r}) .
\end{aligned}
$$


This atom-condensed representation of the electronic structure response is in principle exact in the limit of a complete basis set. Given the atom-centered basis set expansions of eq. (1), the parametrized matrix form of the KS-DFT linear response equation, i.e., the working equation of ACKS2, reads

$$
\begin{array}{r}
\left.\left[\begin{array}{ccc}
{\left[\eta_{n, n^{\prime}}\right]_{\mathrm{N}, \mathrm{N}}} & -\left[O_{n, m}\right]_{\mathrm{N}, \mathrm{M}} & {\left[D_{n, 1}\right]_{\mathrm{N}}} \\
-\left[O_{m, n}\right]_{\mathrm{M}, \mathrm{N}} & {\left[\chi_{m, m^{\prime}}\right]_{\mathrm{M}, \mathrm{M}}} & 0 \\
{\left[D_{1, n}\right]_{\mathrm{N}}} & 0 & 0
\end{array}\right]\left[\begin{array}{c}
{\left[c_{n, 1}\right]_{\mathrm{N}}} \\
{\left[d_{m, 1}\right]_{\mathrm{M}}} \\
\Delta \mu
\end{array}\right]=\begin{array}{c}
{\left[-V_{n, 1}\right]_{\mathrm{N}}} \\
{\left[0_{m, 1}\right]_{\mathrm{M}}} \\
0
\end{array}\right] \\
\mathbf{P x}=-\mathbf{V} .
\end{array}
$$

The right hand side vector with elements $[\mathbf{V}]_{n}=\int d \mathbf{r} g_{n}(\mathbf{r}) \Delta v_{\text {ext }}(\mathbf{r})$ represents the potential $\Delta v_{\text {ext }}$ of an external perturbation acting on the system, such as e.g. an external field. The solution vector $\mathbf{x}=\left\{c_{n}, d_{m}\right\}$, collects the expansion series coefficients for the change in electron density and KS potential. The matrix $\mathbf{P}$ on the left hand side of eq. (2) encodes the reduced KS-DFT ground state response information, condensed according to the choice of basis set in eq. (1). It contains four different parameters, $\left\{\eta_{i, j}, \chi_{i, j}, O_{i, j}, D_{i}\right\}$, which are all well-defined expectation values of the KS-DFT ground state electronic structure.

Two parameters, $\eta_{i, j}$ and $\chi_{i, j}$, require KS-DFT ground state orbitals and energies for their calculation:

$$
\begin{gathered}
\eta_{i, j}=\iint g_{i}(\mathbf{r})\left(\frac{1}{\left|\mathbf{r}-\mathbf{r}^{\prime}\right|}+\frac{\partial^{2} E^{\mathrm{xc}}[\rho]}{\partial \rho(\mathbf{r}) \partial \rho\left(\mathbf{r}^{\prime}\right)}\right) g_{j}\left(\mathbf{r}^{\prime}\right) d \mathbf{r} d \mathbf{r}^{\prime} \\
\chi_{i, j}=\iint h_{i}(\mathbf{r})\left(\frac{\partial^{2} E^{\mathrm{KS}}\left[v_{\mathrm{KS}}\right]}{\partial v_{\mathrm{KS}}(\mathbf{r}) \partial v_{\mathrm{KS}}\left(\mathbf{r}^{\prime}\right)}\right) h_{j}\left(\mathbf{r}^{\prime}\right) d \mathbf{r} d \mathbf{r}^{\prime} .
\end{gathered}
$$

The hardness kernel $\eta_{i, j}$ accounts for the energetic cost of electronic polarization due to changes in the electron density $\Delta \rho$. The non-interacting linear response kernel $\chi_{i, j}$ accounts 
for the energetic cost of electronic polarization due to changes in the Kohn-Sham potential $\Delta v_{\mathrm{KS}}$. The remaining two ACKS2 parameters, $O_{i, j}$ and $D_{i}$, are independent of the KS electronic structure and depend only on the choice of the basis functions in eq. (1). $O_{i, j}$ is the overlap integral of basis functions for density and KS potential response: $O_{i, j}=$ $\int g_{i}(\mathbf{r}) h_{j}(\mathbf{r}) d \mathbf{r}$, accounting for the energetic contribution of concerted changes of electron density and KS potential. $D_{i}=\int g_{i}(\mathbf{r}) d \mathbf{r}$ and ensures the conservation of total charge.

With these parameters, the total ACKS2 polarization energy of the system can be expressed as ${ }^{53}$

$$
\Delta E^{\text {pol. }}=\frac{1}{2} \sum_{n, n^{\prime}}^{N, N} c_{n} \eta_{n, n^{\prime}} c_{n^{\prime}}+\frac{1}{2} \sum_{m, m^{\prime}}^{M, M} d_{m} \chi_{m, m^{\prime}} d_{m^{\prime}}-\sum_{n, m}^{N, M} c_{n} O_{n, m} d_{m}+\sum_{n}^{N} c_{n} D_{n} .
$$

\subsection{Fragment-ACKS2}

We here introduce a fragment-approach to ACKS2, termed fragment-ACKS2 or f-ACKS2. The key idea here is to divide a large condensed phase system into smaller polarizable subunits or fragments, typically molecules, and account for the total electronic polarization in a simulation cell by means of the individual fragment responses. Specifically, in this study the f-ACKS2 technique is applied to evaluate the dielectric response contributions to the intermolecular interactions in dense phase media. Therefore, as illustrated for a benzene molecular dimer in fig. 1, fragments are chosen to represent the entire simulation cell and consecutively parametrized in a vacuum reference framework. This means, the f-ACKS2 matrices for each fragment are calculated individually from the KS-DFT electronic ground state of an isolated molecule in vacuum. Additionally, an effective interaction potential of the isolated ground state charge density of each fragment is developed, which is used as external perturbation to simulate the effect of neighbouring molecules in dense phase media. Solution of the f-ACKS2 equations then yields the electronic polarization of a vacuum-level parametrized fragment embedded in a dense phase environment represented by vacuum-level charge potentials. This way, the f-ACKS2 approach follows the spirit of force field techniques to treat contributions 
f-ACKS2 scheme
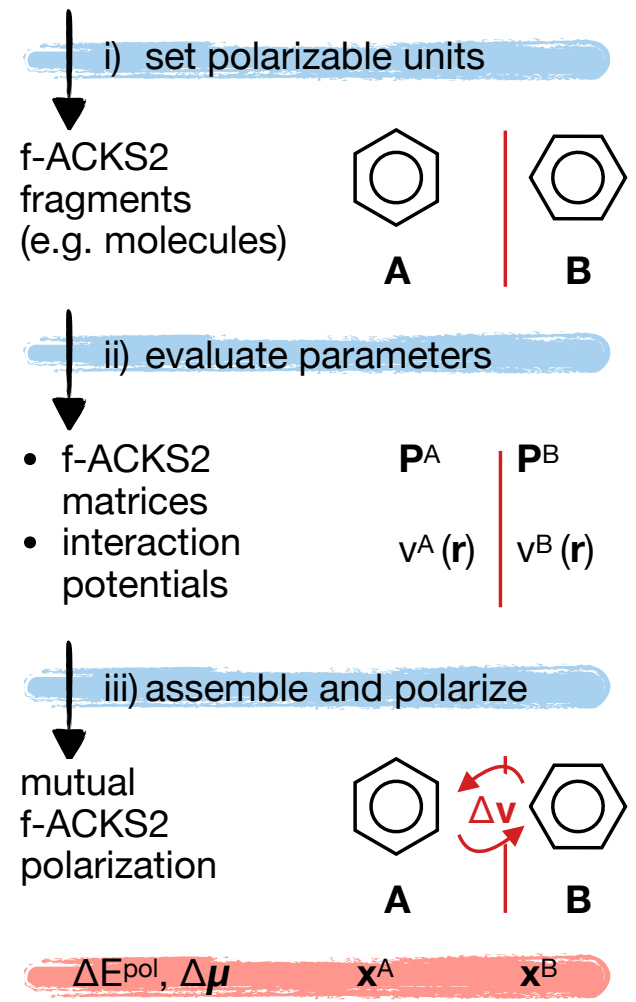

Figure 1: Illustration of the methodological steps of the f-ACKS2 approach for the mutual polarization of two benzene molecules.

to the total energy on different footings based on e.g. chemical connectivity - bonded or nonbonded - or distance measures - long-range vs. short-range. It provides a computationally efficient tool to account for the electronic polarization between fragments, like molecules in the condensed phase, and hence is ideally suited for use in molecular mechanics simulations. In the following, the ACKS2 foundations of the three practical steps-i) fragmentation, ii) parametrization and iii) mutual polarization - depicted in fig. 1 are assessed, starting from the viewpoint of an ACKS2 matrix formulation of the entire simulation cell.

In a nutshell, the system cell fragmentation step is justified by theoretical arguments to decouple the response between different fragments (sparsification), to change the ACKS2 framework from an embedded molecule to a vacuum-level reference electronic structure and to set a suitable charge constraint. The parametrization step is concerned with the calcula- 
tion of the f-ACKS2 matrix elements and an efficient yet accurate inter-fragment interaction potential. The fragment polarization step involves the solution of the f-ACKS2 matrix equations and numerical implementations to allow possible coupling between different fragments, as well as correct evaluation of the response properties.

Step i), the partitioning of a simulation system in $\kappa$ polarizable fragments is a new concept to the ACKS2 theory, inspired by other constraint and fragmentation techniques like fragment-orbital DFT. ${ }^{54}$ Similar schemes have been reported as ad hoc corrections in charge equilibration schemes, where a molecule has been partitioned topologically in small sub-units to avoid the erroneous superlinear scaling of molecular polarizabilities with increasing size. ${ }^{49,55}$ The choice of fragments is in principle arbitrary, e.g. following atom proximity, chemical connectivity or functional grouping. The use of molecules as polarizable sub-units arises naturally in molecular condensed phase system like organic crystals or solvated proteins. The f-ACKS2 technique represents a sparsification approach to the total electronic response, which in the present study contains three conceptual approximations (fragmentation of the ACKS2 equations and methodological implications are derived in detail in the supporting information). First, all ACKS2 matrix elements $\left(O_{i, j}, \eta_{i, j}, \chi_{i, j}\right)$ related to basis functions located on different fragments are set to zero, which prohibits the coupling of the electronic response between different fragments. Note, any intra-fragment matrix elements are unchanged. The overlap matrix elements $O_{i, j}$ (between density basis functions and KS potential functions) generally depend on the radial decay of each function and the distance between them, which leads to small overlaps for well-separated molecular fragments. Following the theorem of Unsöld, ${ }^{56}$ ACKS2 matrix elements of the non-interacting linear response kernel $\chi_{i, j}$ related to different molecular fragments - i.e. at a large distance and small electronic overlap between different atomic sites - are approximately zero. ${ }^{51}$ Similarly, the exchange-correlation contributions to the hardness kernel $\eta_{i, j}$ for semi-local functionals such as $\mathrm{PBE}^{57}$ rapidly decay with increasing interatomic distances, yielding negligible intermolecular contributions to the ACKS2 matrix. Only the classical electrostatic interaction 
contribution to the hardness kernel is long-range in nature and goes well-beyond typical intermolecular distances in dense matter. However, the electronic response in dielectric media (like an organic molecule) to an external potential and especially its induced change of the potential at other fragments is reasonably small, and setting these contributions to zero is a valid first approximation (depending on the system polarizability). The fragmentation (sparsification) and its practical impact on the ACKS2 (intermolecular vs. intramolecular) matrix elements are illustrated for a molecular dimer of benzene, see section 3.1. As model refinement to f-ACKS2, mutual fragment response interactions due to classical electrostatic contributions is implemented in an iterative, self-consistent field approach introduced in section 2.3 .

The second approximation in our fragmentation approach concerns the evaluation of the ACKS2 parameters for the individual fragments. Executing and postprocessing a DFT calculation for the entire simulation cell at every molecular dynamics or Monte Carlo simulation step (and then set all inter-fragment matrix elements to zero anyways) is computationally prohibitive in force field methods. One route to circumvent this is to sample a reasonable number of relevant structures of the molecule embedded in the dense phase environment and extract an estimate of their exactly calculated ACKS2 parameters (e.g. via averaging, interpolation, or even machine-learning). In this study we opt for an even more transferable approach, where the parameters of each fragment are derived from a reference DFT calculation of a single molecule in vacuum. Other fragments otherwise present in dense matter is introduced by an approximate Coloumb response interaction potential (like a simple point charge model), very much in the spirit of other force field techniques. Furthermore, a vaccuum reference parametrized f-ACKS2 model allows the evaluation of electronic polarization contributions to the intermolecular interactions, which will be studied for a set of small (partially charged) aromats below.

The third, and last, approximation concerns the constraint of the total charge due to electronic rearrangemenets present in the ACKS2 method, c.f. last row in eq. (2), which 
necessitates a translation to fragment contributions in the f-ACKS2. Practically, this yields two distinct choices. In a straightforward approach, a constraint is imposed on the overall polarization charge, i.e. summation over all fragment charges is constant, which allows charge-transfer between different sites. Thereby, charge equilibration is achieved in the entire system and the responses of the individual fragments are effectively coupled by chargetransfer energetics (any implementation requires a numerical solution of a coupled set of linear equations). Alternatively, a charge constraint is imposed for each individual fragment in f-ACKS2 preventing charge transfer between different sites. This is the approach taken in this work. While it is expected to be a good approximation for the (adiabatic) ground state of non- or weakly-polar systems, it is the natural choice for the modelling of the charge localized (or (quasi)-diabatic) electronic states of electron transfer reactions ${ }^{58}$ and charge transport in the condensed phase. ${ }^{59-61}$

Step ii), the parametrization process of the f-ACKS2 response matrices follows the standard ACKS2 model for each of the $\kappa$ fragments in vacuum. The perturbation of a given fragment A by the other fragments B is modeled by a sum of fragment potentials $v_{\text {frag }}^{A}$,

$$
\Delta v_{\text {ext }}^{A}(\mathbf{r})=\sum_{B \neq A}^{\kappa} v_{\text {frag }}^{B}(\mathbf{r})
$$

The idea of an effective potential representation $v_{\text {frag }}^{B}$ here is a general concept and can be tuned to specific numerical needs and applications. In order to avoid costly real space integrals in the determination of the perturbation vector $\mathbf{V}$, we chose a discrete representation based on fixed atom-centered point charges (fc), i.e. $v_{\text {frag }}^{B}=v_{\mathrm{fc}}^{B}$. The latter represents a simple Coulomb interaction term, but neglects higher fixed multi-pole terms or exchange-correlation contributions between the fragments. While it would also be possible to introduce, e.g. atom-centered multipoles, to describe fragment-fragment interactions, our benchmark results presented in section 3 show that already such a simple representation is able to capture most of the induced polarization effects.

Step iii), computing the (non-self-consistent) response for each individual fragment again 


\section{(non-self-consistent) f-ACKS2}
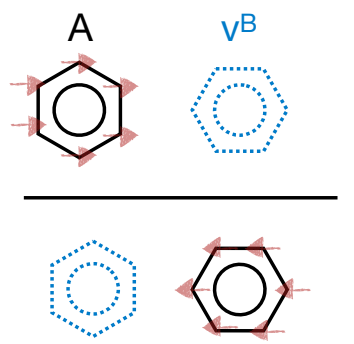

$v^{A}$

interfragment coupling to $\rho_{0}$ self-consistent f-ACKS2

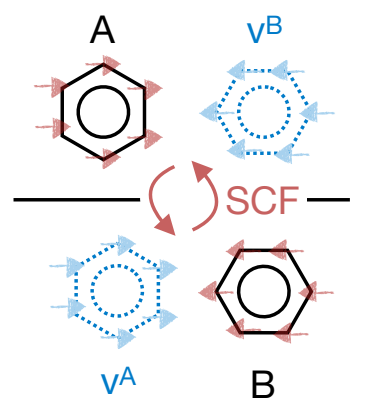

add. interfragment coupling to $\Delta \rho$

Figure 2: Illustration of the effective interaction treatment in f-ACKS2 and scf-ACKS2.

follows standard ACKS2 methodology.

$$
\begin{aligned}
{\left[\mathbf{V}^{A}\right]_{n} } & =\sum_{B \neq A}^{\kappa} \int g_{n}^{A}(\mathbf{r}) v_{\mathrm{fc}}^{B}(\mathbf{r}) d \mathbf{r} \\
\mathbf{P}^{A} \mathbf{X}^{A} & =-\mathbf{V}^{A} .
\end{aligned}
$$

The solution vector $\mathbf{x}^{A}$ contains the expansion coefficients for electron density and KohnSham potential changes, $c_{n}^{A}$ and $d_{m}^{A}$, of basis functions located at fragment A, c.f. eqs. (1a) and (1b). Observables like polarization energy and dipole moment of the individual fragments follow straightforwardly, see. ${ }^{53}$ The overall response is finally obtained by simple superposition of the individual fragment electronic polarization,

$$
\begin{gathered}
\Delta \mu=\sum_{A}^{\kappa} \Delta \mu_{A} \\
\Delta E^{\text {pol. }}=\sum_{A}^{\kappa} \Delta E_{A}^{\text {pol. }} .
\end{gathered}
$$




\subsection{Self-consistent fragment-ACKS2}

With the introduction of a fixed atom-centered point charge representation $v_{\mathrm{fc}}^{B}$, we provide an effective interaction potential between f-ACKS2 fragments based on a simplified electronic ground state charge distribution. However, the influence of mutual electronic polarization interactions between different fragments have not yet been accounted for. Illustrated in fig. 2, the presence of fragment A and its effective ground-state KS potential polarizes fragment B, which in turn induces local potential changes and a response of fragment $\mathrm{A}$, in turn changing the potential on fragment $\mathrm{A}$, and so on. In total, this yields a contribution $v_{\text {resp }}^{B}$ to the local potential of a fragment due to the polarization of all other fragments in the system

$$
\Delta v_{\mathrm{ext}}^{A}(\mathbf{r})=\sum_{B \neq A}^{\kappa} v_{\mathrm{fc}}^{B}(\mathbf{r})+\sum_{B \neq A}^{\kappa} v_{\mathrm{resp}}^{B}(\mathbf{r})
$$

A straightforward way to introduce coupling of the mutual polarization response interactions is the addition of a is the addition of a Coulomb potential due to the response density, $v_{\text {resp }}^{B}=\sum_{n^{\prime}}^{N^{\prime}} c_{n^{\prime}} \int \frac{1}{\left|\mathbf{r}-\mathbf{r}^{\prime}\right|} g_{n^{\prime}}\left(\mathbf{r}^{\prime}\right) d \mathbf{r}^{\prime}$,

$$
\Delta v_{\mathrm{ext}}^{A}=\sum_{B \neq A}^{\kappa} v_{\mathrm{fc}}^{B}(\mathbf{r})+\sum_{B \neq A}^{\kappa} \sum_{n^{\prime}}^{N^{\prime}} c_{n^{\prime}}^{B} \int \frac{1}{\left|\mathbf{r}-\mathbf{r}^{\prime}\right|} g_{n^{\prime}}^{B}\left(\mathbf{r}^{\prime}\right) d \mathbf{r}^{\prime} .
$$

Note that the mutual response interaction term shown here for fragment A includes the fACKS2 expansion coefficients $c_{n^{\prime}}^{B}$ of the other fragments B. Thus, the new working equations cannot simply be solved by a single matrix inversion of $\kappa$ response-independent fragments, as in f-ACKS2, because now, all fragment perturbations include the polarization of each other and the individual fragment coefficients are interdependent. The interdependence of the (atomic) inducible multipole moments leads to a large, yet sparse ACKS2 set of linear equations for the total system (all fragments). The number of equations scales with the number of basis functions and would introduce a considerable bottleneck in calculations on large clusters/condensed phase systems. The blocks on the diagonal represent individual 
fragments (here parametrized from molecules in vacuum). All inter-fragment off-diagonal elements introduce an approximate coupling, see second term in eq. (9), which is long-range in nature due to the $1 / r$ and $1 / r^{3}$ decay of the electrostatic potential of (point) charges and dipoles, respectively, and somewhat reduces the actual sparsity of the ACKS2 equations. The difference between a fully fragmented f-ACKS2 and the coupled working equations is illustrated for a two fragment system in figure 3 .

An exact solution of the sparse polarization matrix is computationally very expensive, instead it is often approximated to arbitrary accuracy following two general concepts (or combinations thereof). In iterative solver techniques, the simulation cell is divided into smaller subunits (with the smallest being an individual site) for which the individual polarization linear equations are solved, similar to the f-ACKS2 approach. The response of the individual sites to an initially estimated charge distribution updates the local electrostatic potential prompting a new response, and so forth until self-consistency is reached. ${ }^{62,63}$ Alternatively, in time-dependent molecular dynamics simulations, the explicit polarization degrees of freedom can be propagated directly from an initial solution by Lagrangian methods, which introduces an additional thermostat for the response. ${ }^{64,65}$ Here, we employ an iterative self-consistency scheme, termed scf-ACKS2, where the response of each fragment is fed step-wise into the external potential of other fragments until self-consistency is reached,

$$
\begin{aligned}
\mathbf{V}^{A,\{i\}} & =\mathbf{V}_{\mathrm{fc}}^{A}+\mathbf{V}_{\text {response }}^{A}\left(\mathbf{x}^{B,\{i-1\}}\right) \\
{\left[V_{\mathrm{fc}}^{A}\right]_{n} } & =\sum_{B \neq A}^{\kappa} \int g_{n}^{A}(\mathbf{r}) v_{\mathrm{fc}}^{B}(\mathbf{r}) d \mathbf{r} \\
{\left[V_{\text {response }}^{A}\right]_{n}\left(\mathbf{x}^{B,\{i-1\}}\right) } & =\sum_{B \neq A}^{\kappa} \sum_{n^{\prime}}^{N^{\prime}} c_{n^{\prime}}^{B,\{i-1\}} \int g_{n}^{A}(\mathbf{r}) \frac{1}{\left|\mathbf{r}-\mathbf{r}^{\prime}\right|} g_{n^{\prime}}^{B^{\prime}}\left(\mathbf{r}^{\prime}\right) d \mathbf{r} d \mathbf{r}^{\prime}
\end{aligned}
$$

The additional superscript $\{i\}$ indicates the step number within the iterative scf-ACKS2 scheme. In the scf-ACKS2 scheme, all interaction integral vectors in $\mathbf{V}_{\mathrm{fc}}$ and $\mathbf{V}_{\text {response }}$ are 


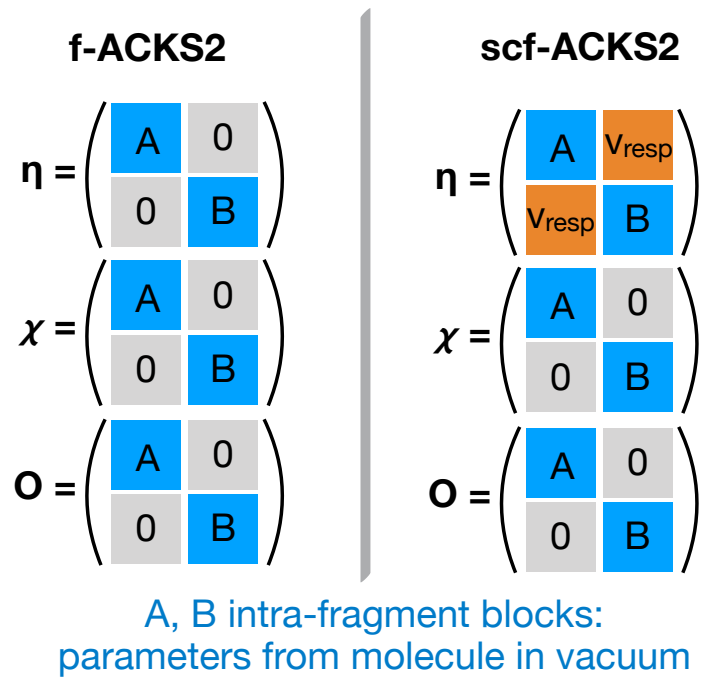

Figure 3: Illustration of the different parameter elements in a direct matrix approach to f-ACKS2 excluding and including the response coupling $v_{\text {resp }}$ (as a function of the charge rearrengments $\Delta \rho$ only) between different fragments for a benzene molecular dimer.

constant and can be stored in memory, whereas the expansion prefactors $\mathbf{x}^{A}\left(\left\{c_{n}^{A}\right\}\right.$ and $\left.\left\{d_{m}^{A}\right\}\right)$ change with each iteration step, accounting for the mutual polarization response interaction. Following the update of the perturbation vectors, solution of the ACKS2 matrix equation for each fragment gives the individual expansion coefficient vectors, $\mathbf{P}^{A} \mathbf{x}^{A,\{i\}}=\mathbf{V}^{A,\{i\}} \forall A \in \kappa$. Note, the solutions to the ACKS2 matrix equation of each fragment are interdependent, as defined in eq. (11c). Efficient implementation strategies of the iterative solvers - including initial guesses to the solution, preconditioning and propagation algorithms, e.g. flavors of Jacobi and conjugate gradient methods - to compute reliable explicit polarization properties at reasonable computational cost is a matter of ongoing research. ${ }^{62,63,66}$ Focusing on a proofof-principle in the present study, we include an intermediate linear mixing step for an update of the ACKS2 density and KS-potential expansion coefficients $\mathbf{x}^{A}$ mediated by a constant mixing parameter $\tau_{\text {mix }}<1$,

$$
\mathbf{x}^{A,\{i\}} \rightarrow \tau_{\text {mix }} \cdot \mathbf{x}^{A,\{i\}}+\left(1-\tau_{\text {mix }}\right) \cdot \mathbf{x}^{A,\{i-1\}}
$$

The iteration of the mutual electronic polarization interaction between different fragments 
is continued until self-consistency is reached, ensured by some termination criterion like the maximum change of coefficients to be smaller than some threshold, $\left(\max _{n}\left(\left\{\mid x_{n}^{A,\{i\}}-\right.\right.\right.$ $\left.\left.\left.x_{n}^{A,\{i-1\}} \mid\right\}\right)<\tau_{\text {threshold }}\right) \forall A$.

The scf-ACKS2 scheme is initiated by solution of the f-ACKS2 equations for each fragment where the external perturbation includes the fixed charge distribution interaction potential $v_{\mathrm{fc}}^{B}$. That is, the expansion coefficients in the potential eq. (10) are set to zero for

the first step of the iterative scheme, $c_{n^{\prime}}^{B,\{0\}}=0$. Hence, the initial polarization of each fragment contains the electronic response due to the interactions with the fixed point charge distribution of all other fragments. In principle, SCF convergence can in some cases further be improved by using a different mixing constant $\tau_{\text {init }} \neq \tau_{\text {mix }}$, for the first few steps. In practice, though, for most systems considered here this proved unnecessary, cf. supplementary material.

The electronic response of the total system is obtained by summing up the polarization energies of the individual fragments, but corrected for the double counting of the mutual response polarization interaction contributions, where $\mathbf{x}$ represents the self-consistent solution in the scf-ACKS2 scheme,

$$
\Delta E_{\mathrm{scf}-\mathrm{ACKS} 2}^{\text {pol. }}=\sum_{A}^{\kappa} \Delta E_{A}^{\mathrm{pol} .}-\frac{1}{2} \sum_{A}^{\kappa} \sum_{B \neq A}^{\kappa} \mathbf{x}^{A} \cdot \mathbf{V}_{\text {response }}^{A}\left(\mathbf{x}^{B}\right) .
$$

\section{Results}

\subsection{Parametrization of f-ACKS2.}

First, we highlight the impact of the above discussed approximate treatment of the f-ACKS2 parameters for a molecular dimer of a benzene taken from a single crystal (Pbca), ${ }^{67}$ where each molecule represents one fragment. In fig. 4, the distributions and distance-dependence of the various matrix elements of the total dimer are color-coded to illustrate the relative importance of the individual contributions. Panel A displays the large difference of the 
exchange-correlation contributions to the hardness, $\eta^{\mathrm{xc}}$, for a semi-local functional (PBE) and a fast decay with inter-atomic distance. In Panel B, the Coulomb hardness distributions, $\eta^{\mathrm{H}}$, of inter- and intra-fragment are of similar shape, but slightly shifted by about half an order of magnitude. The slow relative decay of these matrix elements reflects the (nonnegligible) long-range nature of the classical electrostatic interaction contribution of the response. Panel $\mathrm{C}$ demonstrates similar behavior of the distributions of the non-interacting linear response kernel, $\chi$, with respect to intra- and interfragment contributions. However, there is an extended tail in the intra-fragment distribution about two orders of magnitudes larger than the inter-fragment counter-part, which can be expected to dominate the response contributions. The same is reflected in the rather rapid relative decay of the matrix elements with respect to inter-atomic distances. A similar, but less pronounced effect is visible in the distribution and distance plot of the overlap matrix elements $O$. In fig. 5, the difference of same-fragment ACKS2 matrix elements parametrized from the DFT electronic structure of a molecule embedded in the simulation cell versus the molecule in vacuum is illustrated. While both f-ACKS2 matrix elements exhibit broad distributions, they are at least one order of magnitude smaller than the largest (dominating) values displayed in fig. 5. Note that the slightly different distributions for the matrix elements of fragment A and fragment B are a consequence of the effective overlap of basis functions due to their relative orientation.

\subsection{Electronic polarization in molecular dimers $\mathrm{A}-\mathrm{B}^{+}$}

As a first demonstration, we apply the f-ACKS2 model for the electronic polarization between two charge-constrained molecules. Illustratively, we consider dimers of fairly small organic molecules such as benzene (Pbca), ${ }^{67}$ naphthalin $\left(\mathrm{P} 2_{1} / \mathrm{a}\right),{ }^{68}$ anthracene $\left(\mathrm{P} 22_{1} / \mathrm{a}\right){ }^{69}$ and tetracene $(\mathrm{P} \overline{1}),{ }^{70}$ where the first molecule (fragment $\mathrm{A}$ ) is charge neutral, while the second molecule (fragment B) carries a charge of $+1.0 \mathrm{e}$. The dimer geometries are extracted from their respective single crystal structures, taking into account two or three different next nearest neighbour site dimers. With this, we aim for a realistic test case of the electronic 
polarization of a molecule by an electron-hole constrained to another molecule (and vice versa), as e.g. encountered during charge transport in organic semiconductors.

In a first step, we account for fixed-charge distribution interactions between the fragments and exclude any mutual polarization response interactions. The approximate fixed-charged interaction is represented by atom-centered point charges derived from an electrostatic potential fit of the KS-DFT ground state charge distribution, c.f. fixed point charge potential in eq. (6). The respective data are labelled ACKS2(PC) in fig. 6 and therafter. The full electronic response reference data to validate the ACKS2(PC) simulation setup are obtained from a perturbed KS-DFT calculation of the charge-constrained molecules. Therein, the approximate intermolecular interaction potential comes in two flavors: a full Coulomb potential due to electron density and nuclear cores (DFT(FC)) and an atomic point charge approximation of the latter (DFT(PC)) via electrostatic potential fitting. The former is based on a fragment-orbital (FO) DFT implementation ${ }^{54}$ in the FHI-AIMS code ${ }^{71}$ which provides a tool to get a self-consistent KS-DFT calculation of one molecule in the presence of the exact Coulomb potential of the self-consistent charge distribution of another molecule. Further methodological and numerical details of the DFT validation setup and properties are given in the supporting information. A comparison between the different DFT models, DFT(PC) and $\mathrm{DFT}(\mathrm{FC})$, allows us to gauge the error made by approximating the full Coulomb electrostatic interaction potential by point charges. On the other hand, the difference between $\mathrm{DFT}(\mathrm{PC})$ and ACKS2(PC) is the true test for the accuracy and validity of the ACKS2 eletronic polarization model with respect to the DFT parent method.

Figure 6 illustrates the polarization energies and induced dipole moments for each fragment and provides comparison of the f-ACKS2 response model to the DFT data. Note that the response of fragment B to the non-charged non-polar fragment $\mathrm{A}$ is very small and hence more prone to small numerical inaccuracies due to the f-ACKS2 approximations. Assessment of the polarization of fragment A by the positively charged molecule B is less subject to numerical issues and hence of greater significance here. Generally, the trends of the polar- 
ization energy and the dipole moment agree well for fragment A, despite the different levels of interaction potentials and electronic polarization response representations applied here. Following the individual trends, we can decompose the influence of the different levels of electronic response treatment for these molecular dimer systems. DFT(PC) introduces a point charge simplification to the full Coulomb intermolecular interaction potential in DFT(FC). Hence, the differences between the $\mathrm{DFT}(\mathrm{FC})$ and $\mathrm{DFT}(\mathrm{PC})$ trends, black and blue in fig. 6 , represent an estimate of the validity of the first order multipole analysis fragment interaction representation of the full Coulomb potential. For the small organic molecule dimers in fig. 6, the mean unsigned realtive errors for the response - polarization energy and induced dipole moment - of fragment A by the DFT(PC) model are 19\% and 10\%. Extension of the multipole expansion series by including higher angular momentum terms like dipole moments and quadrupole moments could provide a simple conceptional ansatz to improve the interaction potential for even higher accuracies. ${ }^{72}$ The ACKS2(PC) model introduces an approximate electronic response representation to the full KS-DFT polarization DFT(PC), both considering a point charge intermolecular interaction potential. The trends of the polarization properties from both methods agree well with a slight offset due to underestimation by the ACKS2 approach, yielding a relative mean unsigned error of $24 \%$ and $22 \%$ for the polarization energies and induced dipole moments, respectively. Introduction of a larger or more well trained basis set provides a pathway to improve the current ACKS2 model. Considering fragment $\mathrm{B}$, the $\mathrm{ACKS}(\mathrm{PC})$ response matches the $\mathrm{DFT}(\mathrm{PC})$ polarization quite well, while both partly show significant deviation from DFT(FC). Indeed, condensing the Coulomb interaction potential into atom-centered point charge representations is quite inaccurate for large non-polar molecules and can yield tremendous differences in the local perturbation potentials. $^{73,74}$

So far, we disregarded the mutual interaction between the response densities on each fragment, however it can be crucial in non-polar system with strong electrostatic fields such as e.g. a (partly) localized charge on a neighbouring fragment. Figure 7 illustrates the change 
of electronic polarization by addition of the iterative polarization response interaction of scfACKS2 and its validation reference KS-DFT based on a full Coulomb perturbation potential. Note, the scf-ACKS2 model includes different levels of approximations to the electrostatic intermolecular interaction treatment, a simple point charge representation for the fixed-charge distribution and an ACKS2 basis set dependent representation of the mutual polarization response, c.f. eq. (10). The response property trends of scf-ACKS2 generally exhibit good agreement with the DFT validation data. Considering a self consistent polarization response induces small changes of the polarization energies for fragment A and B, leading to further energetic stabilization of the charge, which is about an order of magnitude smaller compared to the polarization energy due to the fixed charge contribution in f-ACKS2, illustrated earlier in fig. 6. The induced dipole moment of fragment B also increases notably, while it remains approximately constant for fragment A. Fragment A does not undergo significant electronic rearrangements during the iteration process, because the response density on the charged fragment B is very small. In contrast, the local potential changes drastically for fragment B compared to the simple fixed-charge contribution by the neutral fragment $\mathrm{A}$ and hence increases its electronic response significantly compared to fig. 6 . 

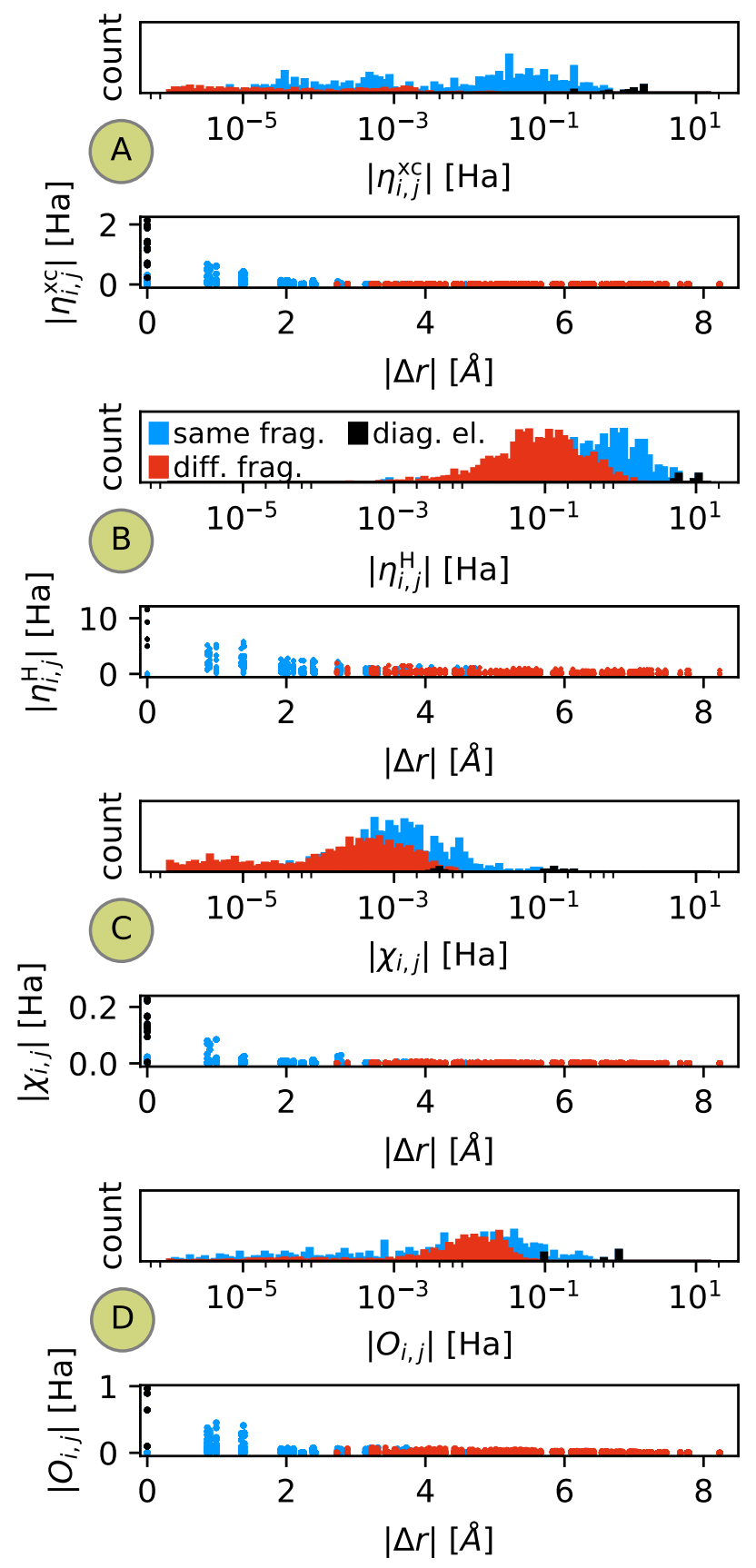

Figure 4: Comparison of inter-fragment and intra-fragment ACKS2 matrix parameters (defined in section 2.1) and their dependence on the distances between the centre of the basis functions for the nearest neighbour benzene molecular dimer of a single crystal. ${ }^{67}$ 


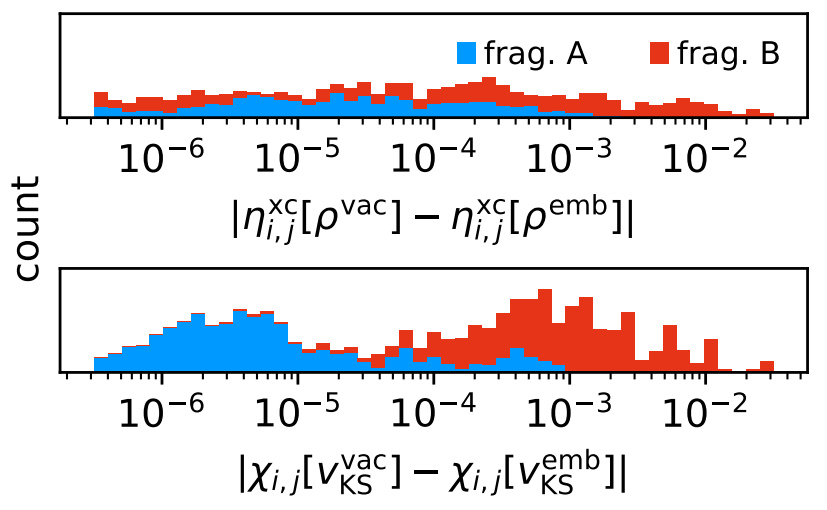

Figure 5: Comparison of intra-fragment f-ACKS2 matrix parameters calculated from the molecule embedded in a dimer (emb) or isolated in vacuum (vac) for a benzene molecular dimer .

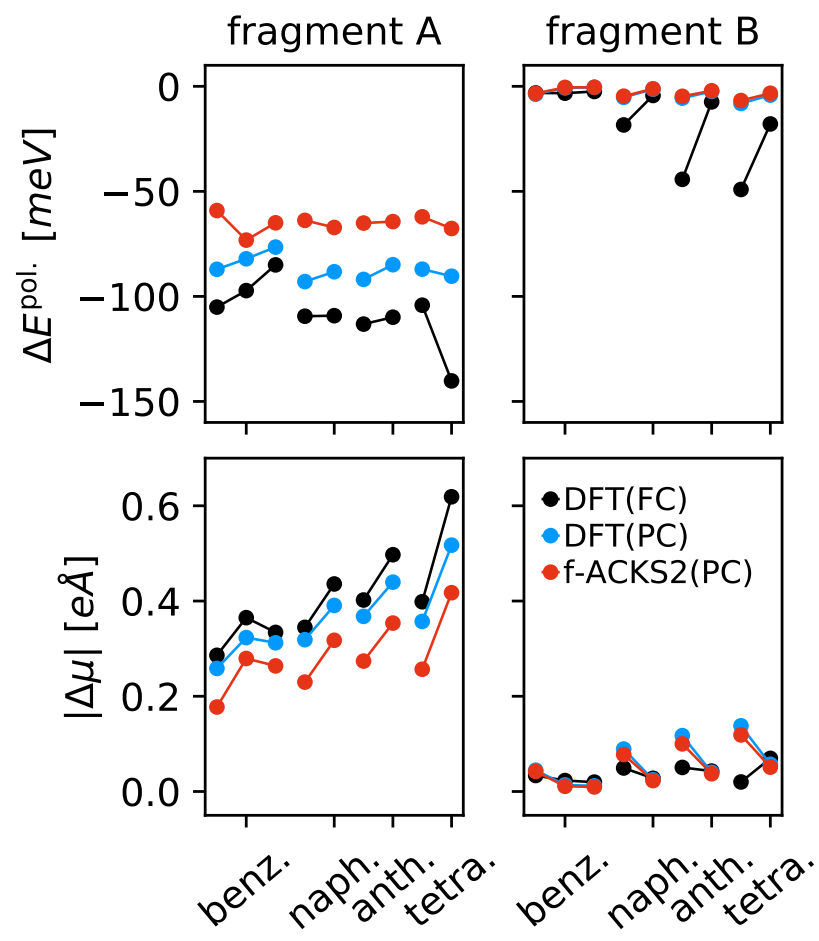

Figure 6: Electronic response for different approximations within the f-ACKS2 approach for a set of small molecular dimers (benzene, naphthalin, anthracene, tetracene), where each datapoint represents a different nearest neighbour geometry extracted from the molecular single crystal structure. The f-ACKS2 polarization energy is calculated according to eq. (8b). See main text and SI for details on DFT calculations. 


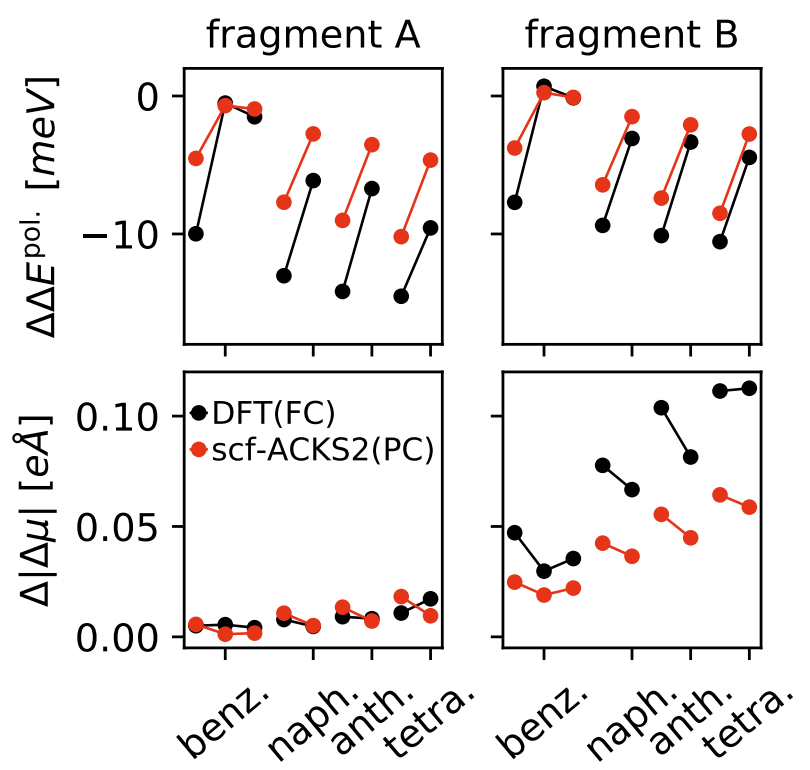

Figure 7: Change of the response by addition of the mutual polarization response interaction potential, $\Delta \Delta E^{\text {pol. }}=\Delta E_{\text {scf-ACKS2 }}^{\text {pol. }}-\Delta E_{\mathrm{f}-\mathrm{ACKS} 2}^{\mathrm{pol}}$ and $\Delta \Delta \mu=\left|\Delta \mu_{\mathrm{scf}-\mathrm{ACKS} 2}-\Delta \mu_{\mathrm{f}-\mathrm{ACKS} 2}\right|$, where $\Delta E_{\mathrm{f}-\mathrm{ACKS} 2}^{\mathrm{pol} \text {. }}$ is calculated according to eq. (8b) and $\Delta E_{\mathrm{scf}-\mathrm{ACKS} 2}^{\mathrm{pol}}$ is calculated according to eq. (13) . The scf-corrections in fragment-based ACKS2 approaches are illustrated for a set of small molecular dimers (benzene, naphthalin, anthracene, tetracene), where each datapoint represents a different nearest neighbour geometry extracted from the molecular single crystal structure. 


\subsection{Electronic polarization in $\mathrm{A}^{+}-\mathrm{B}^{-}$anthracene dimers.}
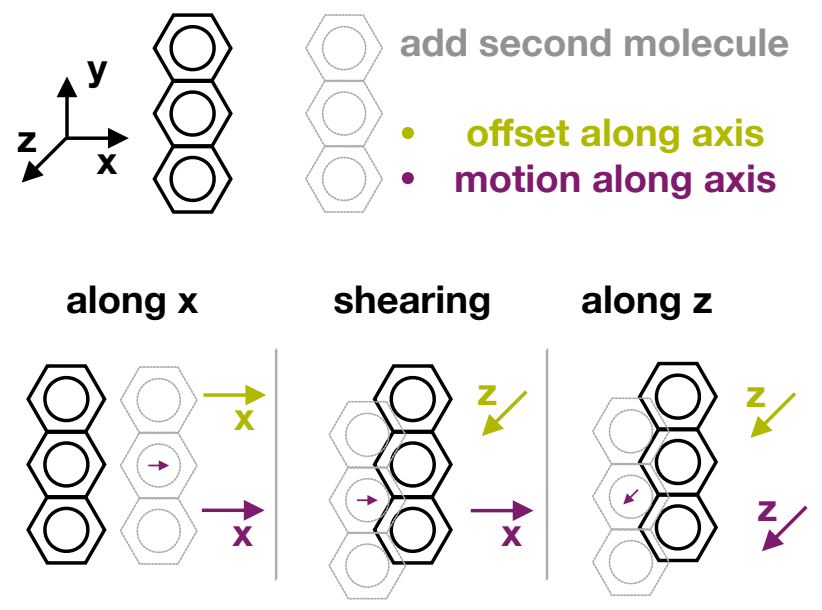

Figure 8: Structure and stacking geometry of anthracene molecular dimers.

We investigate the mutual electronic polarization of two anthracene molecules in different relative orientations and distances, where anthracene molecular fragment $\mathrm{A}$ and $\mathrm{B}$ have a charge of $+1 \mathrm{e}$ and $-1 \mathrm{e}$, respectively. This is a simple model for electronic polarization in an adjacent electron-hole pair or charge transfer exciton. Both anthracene molecules are aligned with their long axes in the y direction, with the second anthracene placed next to the first, shifted along the $x$-, $y$ - or $z$-axis to sample a number of different dimer configurations (cf. fig. 8). The non-self-consistent f-ACKS2 response to a fixed point charge intermolecular interaction model is again validated by $\mathrm{DFT}(\mathrm{PC})$ and $\mathrm{DFT}(\mathrm{FC})$. The response properties, induced dipole moment and polarization energy, are illustrated in fig. 9 for different relative arrangements of the anthracene molecular dimer.

The simulation models for the electronic polarization show good agreement across all different relative arrangements of the anthracene molecular dimer. In fig. 9a, the KS-DFT electronic polarization by the approximate fixed point charge interaction potential, DFT(PC), slightly underestimates (overestimates) the polarization energy of fragment A (fragment B) compared to a full Coulomb representation, DFT(FC), while induced dipole moments exhibit near-perfect coincidence for both methods. Similarly to the previous section, the f-ACKS2 
model tends to underestimate the response properties for all geometries. A more detailed list of the relative deviations, see table 1 , reveals a trend of increasing relative mean unsigned error in the ACKS2 model with rising polarization perpendicular to the molecular bonding plane of the anthracene fragments. We expect the latter two effects to be a consequence of the small ACKS2 basis set representation employed. ${ }^{53}$

We include the mutual polarization interactions between the response of the different fragments to obtain a self-consistent electronic polarization and illustrate the respective change of induced dipole moments and polarization energies in fig. $9 \mathrm{~b}$. The trend of the scf-ACKS2 response changes matches those of the DFT reference very well for the parallel motion along the $x$-axis, while f-ACKS2 - and in consequence the scf-ACKS2 - still struggle to represent the motion along the and $z$-axis and shearing of the molecular dimer. We expect the latter to be a consequence of the underestimation of the response to the fixed-charge interaction in fig. 9a, which accounts for the mutual polarization interaction contributions.

Table 1: Summary of the relative mean unsigned errors for the electronic polarization properties of the $\mathrm{A}^{+}-\mathrm{B}^{-}$anthracene dimers displayed in fig. 9a, calculated following: rmue $_{\mathrm{DFT}(\mathrm{PC})}=\frac{1}{N} \sum_{i}^{N}\left(\frac{x_{i}^{\mathrm{DFT}(\mathrm{PC})}}{x_{i}^{\mathrm{DFT}(\mathrm{FC})}}-1\right)$ and $\operatorname{rmue}_{\mathrm{ACKS} 2(\mathrm{PC})}=\frac{1}{N} \sum_{i}^{N}\left(\frac{x_{i}^{\mathrm{ACKS} 2(\mathrm{PC})}}{x_{i}^{\mathrm{DFT}(\mathrm{PC})}}-1\right)$

\begin{tabular}{cl|cc|cc}
\multicolumn{2}{c|}{ rmue [\%] } & $\Delta \mu_{\mathrm{DFT}(\mathrm{PC})}$ & $\Delta E_{\mathrm{DFT}(\mathrm{PC})}^{\mathrm{pol}}$ & $\Delta \mu_{\mathrm{f}-\mathrm{ACKS}(\mathrm{PC})}$ & $\Delta E_{\mathrm{f}-\mathrm{ACKS} 2(\mathrm{PC})}^{\mathrm{pol} \text {. }}$ \\
\hline \multirow{2}{*}{ along x-axis } & fragment A & 0 & 32 & 13 & 19 \\
& fragment B & 0 & 24 & 19 & 33 \\
\hline \multirow{2}{*}{ shearing } & fragment A & 0 & 14 & 17 & 20 \\
& fragment B & 1 & 24 & 29 & 27 \\
\hline \multirow{2}{*}{ along z-axis } & fragment A & 1 & 16 & 22 & 24 \\
& fragment B & 2 & 50 & 35 & 34
\end{tabular}




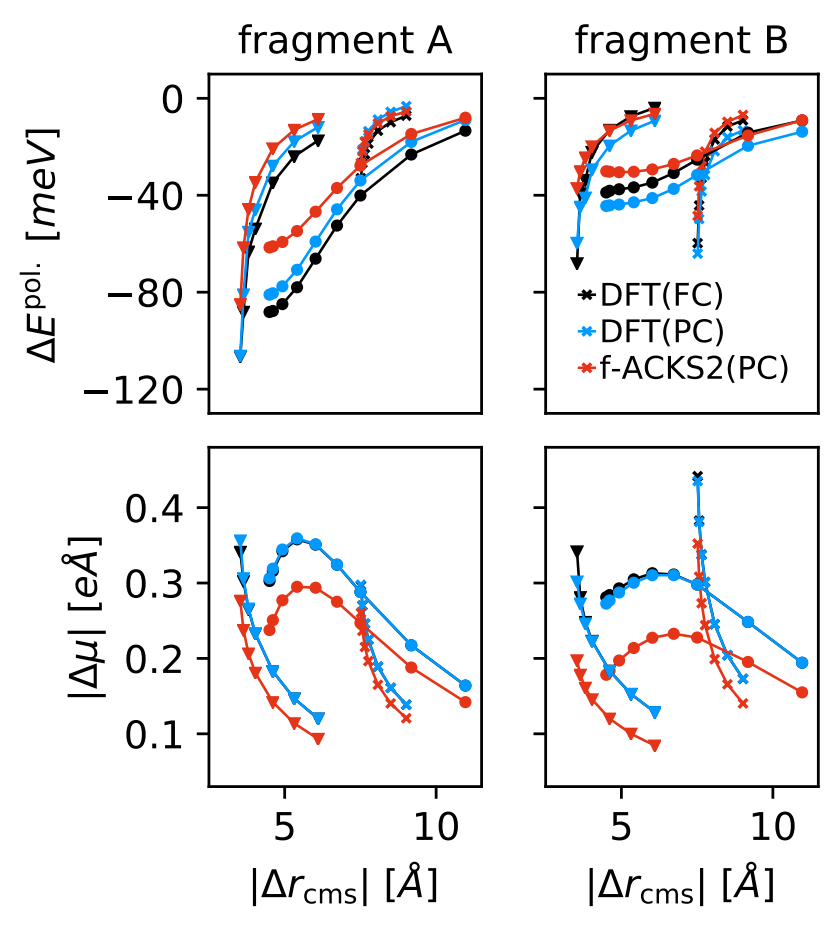

(a) Electronic response to fixed-charge distribution intermolecular interaction.

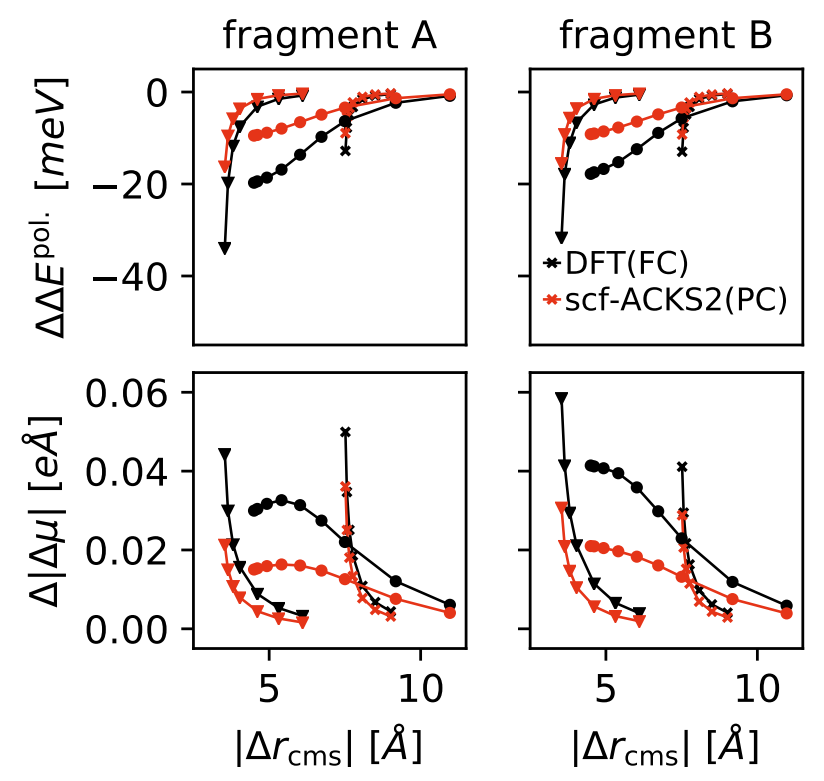

(b) Change of electronic response by addition of the mutual polarization response interaction potential.

Figure 9: Illustration of electronic response for different relative orientations (along $x$-axis: star, shearing: circle, along z-axis: triangle, displayed in fig. 8) and center-of-mass distances $\Delta c m s$ for an $\mathrm{A}^{+}-\mathrm{B}^{-}$anthracene molecular dimer. $\Delta E_{\mathrm{f}-\mathrm{ACKS} 2}^{\text {pol. }}$ is calculated according to eq. (8b), $\Delta E_{\text {scf-ACKS2 }}^{\text {pol. }}$ according to eq. (13). 


\subsection{Polarization energy of electron-hole pair in 1D chain.}
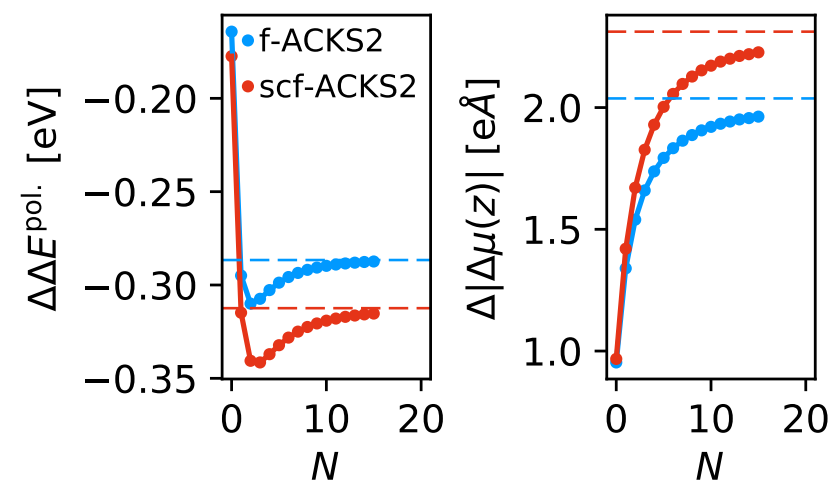

(a) Total electronic response properties of the whole molecular chain. The dashed horizontal lines demonstrate the response properties of two independent chains containing a single independent charge carrier only.

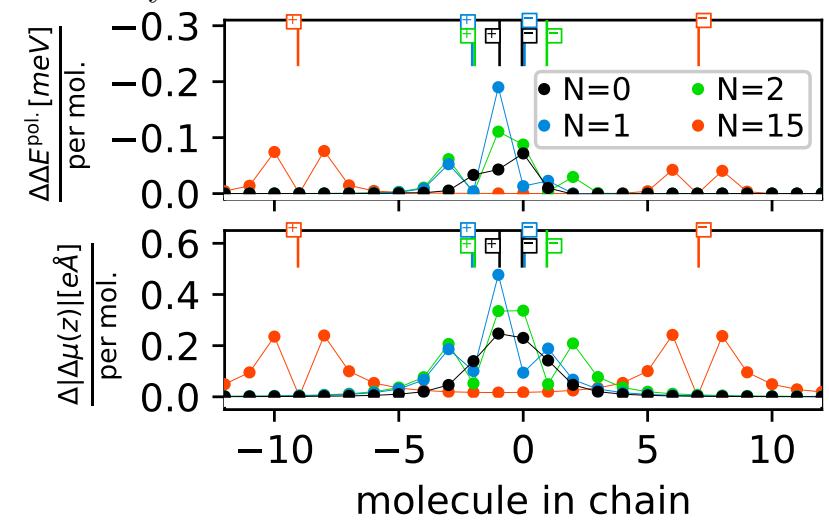

(b) Electronic response properties of the anthracene chain resolved per molecule, where the positions of the charged molecular fragments are indicated individually by the vertical bars on top.

Figure 10: Illustration of the electronic polarization of a linear chain of anthracene molecules containing an electron-hole pair, as a function of the number of separating molecules.

Finally, we study the stabilization of a separated electron-hole pair, located at two different molecular fragments, by the electronic response of its environment. To this end we consider a chain of 50 anthracene molecules with a stacking pattern as shown in fig. 8, "along Z", and a stacking distance of $4.0 \AA$. One molecule of the chain is positively charged and 
another one is negatively charged, all other molecules are neutral, with the number of neutral molecules between the two charged molecules denoted by $N$. Hence, we consider chains of the type $(\mathrm{A})_{M^{-}}(\mathrm{A})^{+1}-(\mathrm{A})_{N^{-}}(\mathrm{A})^{-1}-(\mathrm{A})_{M}$, where $(\mathrm{A})$ denotes an anthracene molecule and $M=\frac{50-N-2}{2}$. The neutral fixed-charge distribution of the chain is again represented by fixed atomic point charges, fitted to the KS-DFT-based electrostatic potential of the free molecules. In order to show screening effects stabilizing the charged molecules we place them at different separations along the chain, starting with a configuration where they are directly adjacent to each other, in analogy to section 3.3. The total electronic response of the anthracene molecule chain is depicted in fig. 10a as a function of $N$. To focus on the polarization effect solely due to the electon-hole pair, we subtract the polarization energy of the chain where all 50 molecules are neutral,

$$
\begin{aligned}
\Delta \Delta E^{\text {pol. }} & =\Delta E^{\text {pol. }}[\text { electron-hole }]-\Delta E^{\text {pol. }}[\text { neutral }] \\
\Delta|\Delta \mu(z)| & =\sum_{A}^{\kappa} \Delta \mid \mu(z)[\text { electron-hole }]\left|-\sum_{A}^{\kappa} \Delta\right| \mu(z)[\text { neutral }] \mid .
\end{aligned}
$$

The total polarization energy as a function of $N$ exhibits a rather unexpected trend. At first, it strongly decreases with increasing $N$, goes through an energy minimum at $N=3$ and then increases to reach a plateau at about $N=12-15$. We explain this observation by two opposing trends. At an initially large distance, both the positively and negatively charged molecule lead to polarization of neutral molecules only, as displayed in fig. 10b for $N=15$, and effectively act like independent charge carriers in a large one-dimensional chain of non-charged anthracene molecules. Once the electron-hole pair separation decreases, here at $N=12-15$, the charge carriers start to polarize each other, while at the same time the polarizability of a negatively (positively) charged anthracene is greater (lesser) than a neutral molecule. In the present one-dimensional chain, the gain in polarization by the electron-carrier outperforms loss in polarization by the hole-carrier and yields a net growth 
in polarization energy with decreasing $N$. On the other hand, several neutral molecules in the chain are exposed to the presence of two charge carriers for small separations of the electron-hole pair. Thereby, the dielectric response of the $N$ intermediate neutral molecules is bumped up linearly due to a simple amplifying superposition of opposite point charge interaction potentials. However, the anthracene molecules outside the electron-hole pair perceive the presence of the latter as finite dipole moment, similar to an ideal point dipole which exhibits stronger locality than an ideal point charge. As $N$ decreases, the dipole perturbation potential contribution increases and polarization energy decreases, which starts to dominate eventually at $N=3$ in the one-dimensional chain here. On the contrary, we find that the absolute value of the induced dipole moment steadily grows with increasing electronhole separation. This effect levels off until it reaches a plateau at $N=15-20$. For large $N$ the polarization energy approaches the polarization energy of two non-interacting charges (dashed line), as it should. The induced dipole moments per anthracene molecule display a larger effective range-dependency, c.f. $N=15$ plot in fig. 10b. Hence, the point-dipolelike contributions to the intermolecular perturbation potential dominate the trend of the total dipole moment and overshadow any polarizability effects of the charged anthracene molecules. Figure 10b thus explains the mismatching trends of the polarization energy (dip at $N=3$ ) and the induced dipole moment (no dip) observed in fig. 10a.

Overall, only a few molecules adjacent to the electron or hole carriers significantly contribute to the $\Delta \Delta E^{\text {pol. }}$ and $\Delta|\Delta \mu(z)|$. This observed locality of the response in even a low dielectric system such as the here treated organic semiconductor chain opens up the f-ACKS2 method for use in efficient force field calculations based e.g. on neighbor lists.

Figure 10a further illustrates the impact of polarization response self-consistency introduced in the scf-ACKS2 approach. As expected, the energetic stabilization and induced dipole moments increase compared to the non-self-consistent treatment. Despite a rather small difference, the non-iterative f-ACKS2 approach predicts the largest polarization stabilization for a spacing of two molecules, while the scf-ACKS2 yields three spacing fragments. 
Finally, in order to gauge the importance of f-ACKS2/scf-ACKS2 polarization compared to the total electrostatic energy, we also need to account for the interaction between the two charge carriers themselves, not currently included in our approach. Instead we compute them with a simple Coulomb model based on our point charge representation of the electrostatic potential. A more detailed description of this approximation can be found in the supporting information. The total electrostatic energy of the molecular chain calculated using this model is depicted in section 3.4. While the total electrostatic interaction energy in this system is dominated by the contributions of the (unpolarized) fixed-charge contribution, the electronic polarization energy as obtained by ACKS2 calculations yields a notable energetic stabilization, particularly at medium to large electron-hole separations. On the other hand, the mutual polarization response as described by our scf-ACKS2 scheme is almost negligible on the energy scales in section 3.4 and may therefore be regarded as a high-accuracy option for specific simulation targets.

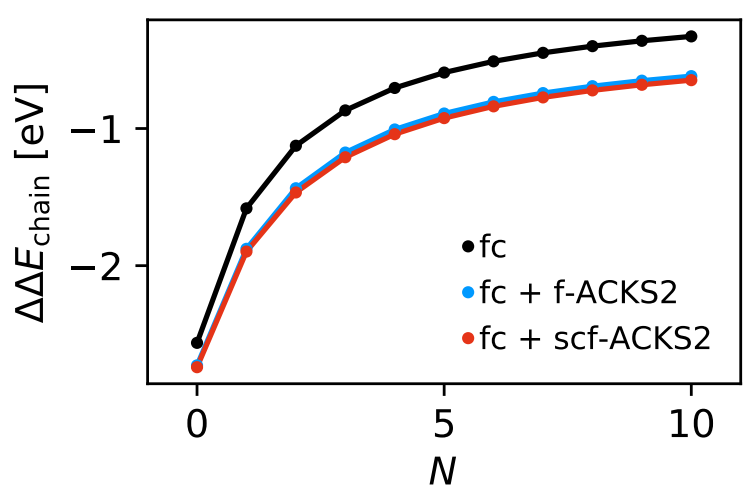

Figure 11: Illustration of the total electrostatic interaction energy of a one-dimensional chain of anthracene molecules based on a simple fixed-charge interaction model (fc) and f-ACKS2/scf-ACKS2 electronic polarization. 


\section{Conclusion}

In summary, here we have introduced a novel fragment-based approach to ACKS2 that allows us to calculate the electron density response, electronic polarization energy and induced dipole moments of molecules in condensed phase molecular systems. We presented a detailed discussion of the fundamental approximations within the f-ACKS2 approach, with particular focus on the applied perturbation treatment, based on fixed-charge distribution interactions. These can optionally be augmented by a self-consistent mutual polarization response contribution. Validation of the electronic polarization contribution from f-ACKS2 and scf-ACKS2 show reasonable accuracy compared to full KS-DFT for different singly-charged organic molecular dimers of as well as various relative orientations of an electron-hole dimer. Already a simple point charge representation of the intermolecular perturbation potential was shown to account for the majority of the molecular polarization. A more accurate representation of the unperturbed electrostatic potential of molecules through inclusion of higher order multipole terms can be expected to further improve the f-ACKS2 electronic response. Additionally, future development of more flexible response basis sets beyond the simple s-p Gaussian basis used here should further improve the accuracy of the ACKS2 method compared to the parent-DFT method.

Finally, a 1D chain of anthracene molecules showcased the ability of the f-ACKS2 and scf-ACKS2 approaches to include first-prinicples-based electronic polarization in large molecular systems at a negligible cost compared to explicit electronic structure calculations. The present work is an important step towards force field development with ACKS2-based electronic polarization.

\section{Methods}

Throughout this study, DFT reference calculations as well as the KS electronic structure dependent ACKS2 parameter evaluations were carried out with the FHI-aims full potential all 
electron DFT simulation package. ${ }^{71}$ We applied the PBE generalized gradient approximated density functional and electron spin was treated explicitly. Integrations were conducted using "tight" integration grids with wave functions expanded in a tier 2 numeric atomic orbital basis, to ensure the numerical convergence of our results.

\section{Contributions}

The initial idea to the project was conceived by J.B. and H.O., all authors contributed to the derivation of the (sc)f-ACKS2 method. P.G. conducted all implementation work and all calculations presented here. P.G. wrote the first draft of the manuscript, later corrected by J.B. and H.O.

\section{Competing Interests}

The authors declare no competing interests.

\section{Data Availability}

Training data for the Gaussian parametrization as well as raw data of the test-sets are available for free at the media server of the Technical University of Munich at the following link https://mediatum.ub.tum.de/1546236.

\section{Code Availability}

All software used in this work is present as an in-house proof-of-concept Python package to be made available upon reasonable request. 


\section{Acknowledgement}

The authors gratefully gratefully acknowledge support from the Solar Technologies Go Hybrid Initiative of the State of Bavaria. P.G. further acknowledges the support of the Technische Universität München - Institute for Advanced Study, funded by the German Excellence Initiative (and the European Union Seventh Framework Programme under Grant Agreement №291763). H.O. acknowledges support from the German Research Foundation (DFG), priority program 1928 COORNETS, Grant OB425/3-1. J.B. acknowledges TUM-IAS for the award of a generous Hans-Fischer Fellowship.

\section{Supporting Information Available}

\section{References}

(1) Plimpton, S. Fast Parallel Algorithms for Short-Range Molecular Dynamics. Journal of Computational Physics 1995, 117, 1-19.

(2) Sun, H. COMPASS: An ab Initio Force-Field Optimized for Condensed-Phase ApplicationsOverview with Details on Alkane and Benzene Compounds. The Journal of Physical Chemistry B 1998, 102, 7338-7364.

(3) Brooks, B. R.; Brooks III, C. L.; Mackerell Jr., A. D.; Nilsson, L.; Petrella, R. J.; Roux, B.; Won, Y.; Archontis, G.; Bartels, C.; Boresch, S.; Caflisch, A.; Caves, L.; Cui, Q.; Dinner, A. R.; Feig, M.; Fischer, S.; Gao, J.; Hodoscek, M.; Im, W.; Kuczera, K.; Lazaridis, T.; Ma, J.; Ovchinnikov, V.; Paci, E.; Pastor, R. W.; Post, C. B.; Pu, J. Z.; Schaefer, M.; Tidor, B.; Venable, R. M.; Woodcock, H. L.; Wu, X.; Yang, W.; York, D. M.; Karplus, M. CHARMM: The biomolecular simulation program. Journal of Computational Chemistry 2009, 30, 1545-1614.

(4) Senftle, T. P.; Hong, S.; Islam, M. M.; Kylasa, S. B.; Zheng, Y.; Shin, Y. K.; Junker- 
meier, C.; Engel-Herbert, R.; Janik, M. J.; Aktulga, H. M.; Verstraelen, T.; Grama, A.; van Duin, A. C. T. The ReaxFF reactive force-field: development, applications and future directions. npj Computational Materials 2016, 2, 15011.

(5) Mackerell Jr., A. D. Empirical force fields for biological macromolecules: Overview and issues. Journal of Computational Chemistry 2004, 25, 1584-1604.

(6) Vanommeslaeghe, K.; Hatcher, E.; Acharya, C.; Kundu, S.; Zhong, S.; Shim, J.; Darian, E.; Guvench, O.; Lopes, P.; Vorobyov, I.; et al., CHARMM general force field: A force field for drug-like molecules compatible with the CHARMM all-atom additive biological force fields. Journal of Computational Chemistry 2009, NA-NA.

(7) Futera, Z.; Blumberger, J. Adsorption of Amino Acids on Gold: Assessing the Accuracy of the GolP-CHARMM Force Field and Parametrization of Au-S Bonds. Journal of Chemical Theory and Computation 2019, 15, 613-624.

(8) Mueller, J. E.; van Duin, A. C. T.; Goddard, W. A. Development and Validation of ReaxFF Reactive Force Field for Hydrocarbon Chemistry Catalyzed by Nickel. The Journal of Physical Chemistry C 2010, 114, 4939-4949.

(9) Thiel, W. Computational Catalysis - Past, Present, and Future. Angewandte Chemie International Edition 2014, 53, 8605-8613.

(10) Yun, K.-S.; Pai, S. J.; Yeo, B. C.; Lee, K.-R.; Kim, S.-J.; Han, S. S. Simulation Protocol for Prediction of a Solid-Electrolyte Interphase on the Silicon-based Anodes of a Lithium-Ion Battery: ReaxFF Reactive Force Field. The Journal of Physical Chemistry Letters 2017, 8, 2812-2818.

(11) Heenen, H. H.; Voss, J.; Scheurer, C.; Reuter, K.; Luntz, A. C. Multi-ion Conduction in Li3OCl Glass Electrolytes. The Journal of Physical Chemistry Letters 2019, 10, 2264-2269. 
(12) Rühle, V.; Lukyanov, A.; May, F.; Schrader, M.; Vehoff, T.; Kirkpatrick, J.; Baumeier, B.; Andrienko, D. Microscopic Simulations of Charge Transport in Disordered Organic Semiconductors. Journal of Chemical Theory and Computation 2011, 7, $3335-3345$.

(13) Giannini, S.; Carof, A.; Blumberger, J. Crossover from Hopping to Band-Like Charge Transport in an Organic Semiconductor Model: Atomistic Nonadiabatic Molecular Dynamics Simulation. The Journal of Physical Chemistry Letters 2018, 9, 3116-3123.

(14) Engkvist, O.; Åstrand, P.-O.; Karlström, G. Accurate Intermolecular Potentials Obtained from Molecular Wave Functions: Bridging the Gap between Quantum Chemistry and Molecular Simulations. Chemical Reviews 2000, 100, 4087-4108.

(15) Wang, J.; Wolf, R. M.; Caldwell, J. W.; Kollman, P. A.; Case, D. A. Development and testing of a general amber force field. Journal of Computational Chemistry 2004, 25, $1157-1174$.

(16) Vizcarra, C. L.; Mayo, S. L. Electrostatics in computational protein design. Current Opinion in Chemical Biology 2005, 9, 622-626.

(17) Wen, S.; Beran, G. J. O. Accurate Molecular Crystal Lattice Energies from a Fragment QM/MM Approach with On-the-Fly Ab Initio Force Field Parametrization. Journal of Chemical Theory and Computation 2011, 7, 3733-3742.

(18) Kordt, P.; van der Holst, J. J. M.; Al Helwi, M.; Kowalsky, W.; May, F.; Badinski, A.; Lennartz, C.; Andrienko, D. Modeling of Organic Light Emitting Diodes: From Molecular to Device Properties. Advanced Functional Materials 2015, 25, 1955-1971.

(19) Sherrill, C. D.; Sumpter, B. G.; Sinnokrot, M. O.; Marshall, M. S.; Hohenstein, E. G.; Walker, R. C.; Gould, I. R. Assessment of standard force field models against highquality ab initio potential curves for prototypes of pi-pi, $\mathrm{CH} / \mathrm{pi}$, and $\mathrm{SH} / \pi$ interactions. Journal of Computational Chemistry 2009, 30, 2187-2193. 
(20) Leontyev, I.; Stuchebrukhov, A. Accounting for electronic polarization in nonpolarizable force fields. Phys. Chem. Chem. Phys. 2011, 13, 2613-2626.

(21) Ponder, J. W.; Wu, C.; Ren, P.; Pande, V. S.; Chodera, J. D.; Schnieders, M. J.; Haque, I.; Mobley, D. L.; Lambrecht, D. S.; DiStasio, R. A.; Head-Gordon, M.; Clark, G. N. I.; Johnson, M. E.; Head-Gordon, T. Current Status of the AMOEBA Polarizable Force Field. The Journal of Physical Chemistry B 2010, 114, 2549-2564.

(22) Kramer, C.; Spinn, A.; Liedl, K. R. Charge Anisotropy: Where Atomic Multipoles Matter Most. Journal of Chemical Theory and Computation 2014, 10, 4488-4496.

(23) Jakobsen, S.; Bereau, T.; Meuwly, M. Multipolar Force Fields and Their Effects on Solvent Dynamics around Simple Solutes. The Journal of Physical Chemistry B 2015, 119, 3034-3045.

(24) Cardamone, S.; Hughes, T. J.; Popelier, P. L. A. Multipolar electrostatics. Phys. Chem. Chem. Phys. 2014, 16, 10367-10387.

(25) Gresh, N.; Cisneros, G. A.; Darden, T. A.; Piquemal, J.-P. Anisotropic, Polarizable Molecular Mechanics Studies of Inter- and Intramolecular Interactions and LigandMacromolecule Complexes. A Bottom-Up Strategy. Journal of Chemical Theory and Computation 2007, 3, 1960-1986.

(26) Gordon, M. S.; Smith, Q. A.; Xu, P.; Slipchenko, L. V. Accurate First Principles Model Potentials for Intermolecular Interactions. Annual Review of Physical Chemistry 2013, $64,553-578$.

(27) Shi, Y.; Xia, Z.; Zhang, J.; Best, R.; Wu, C.; Ponder, J. W.; Ren, P. Polarizable Atomic Multipole-Based AMOEBA Force Field for Proteins. Journal of Chemical Theory and Computation 2013, 9, 4046-4063. 
(28) Halgren, T. A.; Damm, W. Polarizable force fields. Curr Opin Struct Biol 2001, 11, $236-42$.

(29) Warshel, A.; Kato, M.; Pisliakov, A. V. Polarizable Force Fields: History, Test Cases, and Prospects. Journal of Chemical Theory and Computation 2007, 3, 2034-2045.

(30) Lopes, P. E. M.; Huang, J.; Shim, J.; Luo, Y.; Li, H.; Roux, B.; MacKerell, A. D. Polarizable Force Field for Peptides and Proteins Based on the Classical Drude Oscillator. Journal of Chemical Theory and Computation 2013, 9, 5430-5449.

(31) Liu, Z.; Timmermann, J.; Reuter, K.; Scheurer, C. Benchmarks and Dielectric Constants for Reparametrized OPLS and Polarizable Force Field Models of Chlorinated Hydrocarbons. The Journal of Physical Chemistry B 2018, 122, 770-779.

(32) Lamoureux, G.; Roux, B. Modeling induced polarization with classical Drude oscillators: Theory and molecular dynamics simulation algorithm. The Journal of Chemical Physics 2003, 119, 3025-3039.

(33) Lemkul, J. A.; Huang, J.; Roux, B.; MacKerell, A. D. An Empirical Polarizable Force Field Based on the Classical Drude Oscillator Model: Development History and Recent Applications. Chemical Reviews 2016, 116, 4983-5013.

(34) Applequist, J. An atom dipole interaction model for molecular optical properties. Accounts of Chemical Research 1977, 10, 79-85.

(35) Davis, M. E.; McCammon, J. A. Electrostatics in biomolecular structure and dynamics. Chemical Reviews 1990, 90, 509-521.

(36) Anisimov, V. M.; Vorobyov, I. V.; Roux, B.; MacKerell, A. D. Polarizable Empirical Force Field for the Primary and Secondary Alcohol Series Based on the Classical Drude Model. Journal of Chemical Theory and Computation 2007, 3, 1927-1946. 
(37) Yu, W.; Lopes, P. E. M.; Roux, B.; MacKerell, A. D. Six-site polarizable model of water based on the classical Drude oscillator. The Journal of Chemical Physics 2013, 138, 034508 .

(38) Xie, W.; Gao, J. Design of a Next Generation Force Field: The X-POL Potential. Journal of Chemical Theory and Computation 2007, 3, 1890-1900.

(39) Gao, J. Toward a Molecular Orbital Derived Empirical Potential for Liquid Simulations. The Journal of Physical Chemistry B 1997, 101, 657-663.

(40) Gao, J. A molecular-orbital derived polarization potential for liquid water. The Journal of Chemical Physics 1998, 109, 2346-2354.

(41) Jiang, N.; Ma, J. Conformational Simulations of Aqueous Solvated alpha-Conotoxin GI and Its Single Disulfide Analogues Using a Polarizable Force Field Model. The Journal of Physical Chemistry A 2008, 112, 9854-9867.

(42) Zhu, Q.; Lu, Y.; He, X.; Liu, T.; Chen, H.; Wang, F.; Zheng, D.; Dong, H.; Ma, J. Entropy and polarity control the partition and transportation of drug-like molecules in biological membrane. Sci. Rep. 2017, 7, 1-10.

(43) Xie, W.; Orozco, M.; Truhlar, D. G.; Gao, J. X-Pol Potential: An Electronic StructureBased Force Field for Molecular Dynamics Simulation of a Solvated Protein in Water. Journal of Chemical Theory and Computation 2009, 5, 459-467.

(44) Jiang, N.; Ma, J. Multi-layer coarse-graining polarization model for treating electrostatic interactions of solvated alpha-conotoxin peptides. The Journal of Chemical Physics 2012, 136, 134105.

(45) Wang, X.; Yan, T.; Ma, J. Polarizable force fields based on physical models and quantum chemical calculations. International Journal of Quantum Chemistry 2015, 115, 545-549. 
(46) Mortier, W. J.; Van Genechten, K.; Gasteiger, J. Electronegativity equalization: application and parametrization. J. Am. Chem. Soc. 1985, 107, 829-835.

(47) Rappe, A. K.; Goddard, W. A. Charge equilibration for molecular dynamics simulations. J. Phys. Chem. 1991, 95, 3358-3363.

(48) York, D. M.; Yang, W. A chemical potential equalization method for molecular simulations. J. Chem. Phys. 1996, 104, 159-172.

(49) Chelli, R.; Procacci, P.; Righini, R.; Califano, S. Electrical response in chemical potential equalization schemes. J. Chem. Phys. 1999, 111, 8569-8575.

(50) Nistor, R. A.; Polihronov, J. G.; Müser, M. H.; Mosey, N. J. A generalization of the charge equilibration method for nonmetallic materials. J. Chem. Phys. 2006, 125, 094108 .

(51) Verstraelen, T.; Ayers, P. W.; Speybroeck, V. V.; Waroquier, M. ACKS2: Atomcondensed Kohn-Sham DFT approximated to second order. J. Chem. Phys. 2013, 138, 074108 .

(52) Verstraelen, T.; Vandenbrande, S.; Ayers, P. W. Direct computation of parameters for accurate polarizable force fields. J. Chem. Phys. 2014, 141, 194114.

(53) Gütlein, P.; Lang, L.; Reuter, K.; Blumberger, J.; Oberhofer, H. Toward FirstPrinciples-Level Polarization Energies in Force Fields: A Gaussian Basis for the AtomCondensed Kohn-Sham Method. Journal of Chemical Theory and Computation 2019, $15,4516-4525$.

(54) Schober, C.; Reuter, K.; Oberhofer, H. Critical analysis of fragment-orbital DFT schemes for the calculation of electronic coupling values. The Journal of Chemical Physics 2016, 144, 054103. 
(55) Lee Warren, G.; Davis, J. E.; Patel, S. Origin and control of superlinear polarizability scaling in chemical potential equalization methods. The Journal of Chemical Physics 2008, 128, 144110.

(56) Ángyán, J. G. Correlation of bond orders and softnesses. Journal of Molecular Structure: THEOCHEM 2000, 501-502, 379-388.

(57) Perdew, J. P.; Burke, K.; Ernzerhof, M. Generalized Gradient Approximation Made Simple. Phys. Rev. Lett. 1996, 77, 3865-3868.

(58) Oberhofer, H.; Blumberger, J. Insight into the Mechanism of the Ru2+-Ru3+ Electron Self-Exchange Reaction from Quantitative Rate Calculations. Angewandte Chemie International Edition 2010, 49, 3631-3634.

(59) Blumberger, J.; McKenna, K. P. Constrained density functional theory applied to electron tunnelling between defects in MgO. Phys. Chem. Chem. Phys. 2013, 15, 21842196.

(60) Spencer, J.; Gajdos, F.; Blumberger, J. FOB-SH: Fragment orbital-based surface hopping for charge carrier transport in organic and biological molecules and materials. The Journal of Chemical Physics 2016, 145, 064102.

(61) Giannini, S.; Carof, A.; Ellis, M.; Yang, H.; Ziogos, O. G.; Ghosh, S.; Blumberger, J. Quantum localization and delocalization of charge carriers in organic semiconducting crystals. Nature Communications 2019, 10, 3843.

(62) Lipparini, F.; Lagardère, L.; Stamm, B.; Cancès, E.; Schnieders, M.; Ren, P.; Maday, Y.; Piquemal, J.-P. Scalable Evaluation of Polarization Energy and Associated Forces in Polarizable Molecular Dynamics: I. Toward Massively Parallel Direct Space Computations. Journal of Chemical Theory and Computation 2014, 10, 1638-1651. 
(63) Nocito, D.; Beran, G. J. O. Massively Parallel Implementation of Divide-and-Conquer Jacobi Iterations Using Particle-Mesh Ewald for Force Field Polarization. Journal of Chemical Theory and Computation 2018, 14, 3633-3642.

(64) Albaugh, A.; Demerdash, O.; Head-Gordon, T. An efficient and stable hybrid extended Lagrangian/self-consistent field scheme for solving classical mutual induction. The Journal of Chemical Physics 2015, 143, 174104.

(65) Vitale, V.; Dziedzic, J.; Albaugh, A.; Niklasson, A. M. N.; Head-Gordon, T.; Skylaris, C.-K. Performance of extended Lagrangian schemes for molecular dynamics simulations with classical polarizable force fields and density functional theory. The Journal of Chemical Physics 2017, 146, 124115.

(66) Aviat, F.; Lagardère, L.; Piquemal, J.-P. The truncated conjugate gradient (TCG), a non-iterative/fixed-cost strategy for computing polarization in molecular dynamics: Fast evaluation of analytical forces. The Journal of Chemical Physics 2017, 147, 161724.

(67) Budzianowski, A.; Katrusiak, A. Pressure-frozen benzene I revisited. Acta Crystallographica Section B 2006, 62, 94-101.

(68) V.I.Ponomarev, L., O.S.Filipenko Kristallografiya 1976, 21, 392.

(69) Brock, C. P.; Dunitz, J. D. Temperature dependence of thermal motion in crystalline anthracene. Acta Crystallographica Section B 1990, 46, 795-806.

(70) Holmes, D.; Kumaraswamy, S.; Matzger, A. J.; Vollhardt, K. P. C. On the Nature of Nonplanarity in the [N]Phenylenes. Chemistry -A European Journal 1999, 5, 33993412.

(71) Blum, V.; Gehrke, R.; Hanke, F.; Havu, P.; Havu, V.; Ren, X.; Reuter, K.; Schef- 
fler, M. Ab initio molecular simulations with numeric atom-centered orbitals. Comp. Phys. Commun. 2009, 180, 2175-2196.

(72) Stone, A. J. Distributed multipole analysis, or how to describe a molecular charge distribution. Chemical Physics Letters 1981, 83, 233-239.

(73) Price, S. L. A distributed multipole analysis of the charge densities of some aromatic hydrocarbons. Chemical Physics Letters 1985, 114, 359-364.

(74) Fowler, P. W.; Buckingham, A. D. Central or distributed multipole moments? Electrostatic models of aromatic dimers. Chemical Physics Letters 1991, 176, 11-18. 


\section{Graphical TOC Entry}

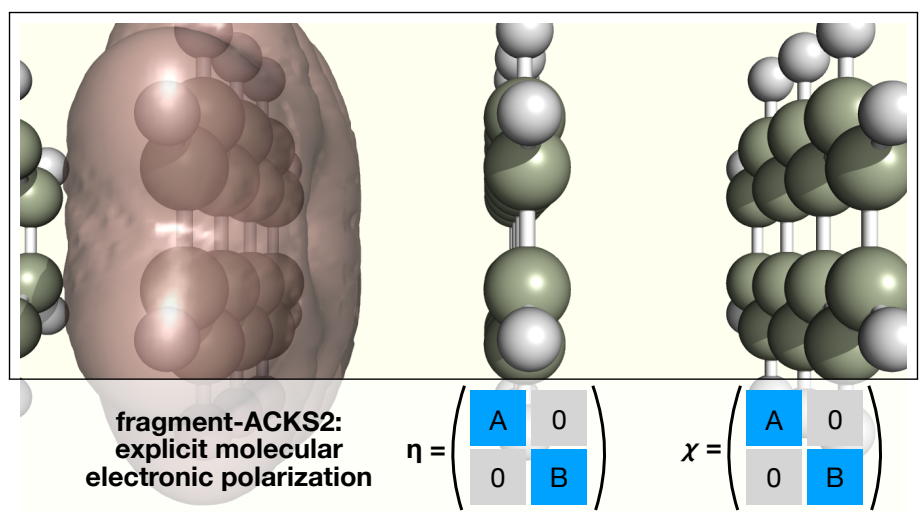

